\title{
Quality of Life and Psychological Distress of Lung Cancer Patients Undergoing Chemotherapy
}

\author{
Paraskevi Prapa ${ }^{\mathrm{a}}$, Ioanna V. Papathanasiou ${ }^{\mathrm{b}, \mathrm{c}}$, Vissarion Bakalis ${ }^{\mathrm{b}}$, Foteini Mallib, \\ Dimitrios Papagiannis ${ }^{b}$, Evangelos C. Fradelos ${ }^{b}$
}

\begin{abstract}
Background: Patients with lung cancer often experience multiple symptoms associated with both the disease itself and the treatment. The disease and therapy-related adverse effects may lead to poor quality of life (QoL) and increased psychological distress. The aim of this study was to investigate the QoL and psychological distress of patients with lung cancer. The relationship between these two aspects was also an area of focus.
\end{abstract}

Methods: This was a quantitative descriptive study. Data collection was done using a self-complementary tool. The data were collected between February and March 2020. The sample consisted of 135 patients with lung cancer who were undergoing chemotherapy in 1-day clinic in Athens (a sample of convenience).

Results: Regarding the QoL of our sample, we observed that the mean score of the physical health component of SF-12 was 38.17 \pm 9.94 and of the mental health component was $45.63 \pm 11.80$. As regards the psychological distress of our sample, we observed that the mean score for depression was $4.55 \pm 5.04$, for anxiety was $3.84 \pm$ 4.17 and for stress was $5.21 \pm 5.01$.

Conclusion: As is clear from the results, lung cancer patients reported poor QoL and increased rates of psychological distress.

Keywords: Quality of life; Psychological distress; Lung cancer; Chemotherapy

\section{Introduction}

Lung cancer is the most common cancer type in the world; more than 2 million cases are diagnosed annually. The increasing in-

Manuscript submitted March 11, 2021, accepted April 20, 2021

Published online May 14, 2021

aAthens Hospital for Chest Diseases "Sotiria", Athens, Greece

bFaculty of Nursing, University of Thessaly, Larissa, Greece

${ }^{c}$ Corresponding Author: Ioanna V. Papathanasiou, Faculty of Nursing, University of Thessaly, Larissa, Greece. Email: iopapathanasiou@yahoo.gr

doi: https://doi.org/10.14740/wjon1371 cidence of disease is reflected by the increasing incidence of mortality [1]. Non-small cell lung malignancy accounts for 80$85 \%$ of all lung cancer cases and is responsible for the majority of the deaths related to lung cancer [2]. Lung cancer is unfortunately considered incurable in cases of advanced diagnosis of the disease [3], while the first-line treatment for patients with advanced non-small cell lung cancer depends on the presence of genetic aberrations (e.g., epidermal growth factor receptor (EGFR) mutations), which are also found in only $10-20 \%$ patients. For other patients, treatment options are limited to the application of platinum-based cytotoxic chemotherapy, whose response rates range from $15 \%$ to $30 \%$ [4]. Patients with smallcell lung cancer, which accounts for $10-15 \%$ of the primary pulmonary malignancies, are almost exclusively smokers, and this disease is characterized by rapid doubling time, high response to first-line chemotherapy and a high relapse rate [5].

Patients with lung cancer often experience multiple symptoms associated with both the disease itself and the treatment, which can independently predict the changes in a patient's functionality as well as treatment failures and post-therapeutic effects. Compared to the discomfort resulting from other types of cancer, lung cancer-related discomfort has been reported to be the most pronounced. The symptoms of the sufferers impose a great burden on their quality of life (QoL) and have serious secondary effects on their emotional, social, physical and mental well-being [6]. QoL is regarded as a multidimensional concept involving physical, functional, psychosocial and spiritual aspects [7].

There are indications that chemotherapy offers benefits to patients with advanced lung cancer in terms of their survival and QoL. In particular, it has been associated with more favorable outcomes such as better QoL and improved symptom control. Even in studies that did not primarily mention a more favorable effect of chemotherapy on the QoL, patients have reported positive effects of chemotherapy on particular areas as well as on functional activity and pain [8]. Various factors appear to affect the QoL of patients with lung cancer [9]. Psychological discomfort is associated with reduced functionality and reduced QoL in terminal-stage patients [10]. The accompanying psychological symptoms of depression, indifference to the future and suicidal behaviors are often present during diagnosis and disease [9]. Studies have reported high incidence of depression (33-44\%) in lung cancer patients $(9.11 \%)$.

Health-related QoL can be a useful measure for assessing the impact of therapeutic processes on patients' holistic 
approach to life [11]. According to Wilson and Cleary, healthrelated QoL refers to the satisfaction of an individual with the parameters of his life at the level affected by health. They also added that it deals mainly with those factors that fall within the scope of healthcare systems and healthcare providers [12]. Individuals facing chronic and life-threating conditions report poor health and decreased QoL [13]. Research on the QoL of patients with lung cancer [9] has also found the effects of the symptoms of treatments on QoL and depression $[9,14,15]$. Although many studies have been carried out on QoL and mental health internationally with patients having lung cancer, research in this field is limited in Greece [16].

The aim of this study was to investigate the QoL and mental stress of patients with lung cancer as well as the relationship between them.

\section{Materials and Methods}

This was a quantitative descriptive study. Data collection was done using a self-complementary tool. The data were collected between February and March 2020. The sample consisted of 135 patients with lung cancer who were undergoing chemotherapy in 1-day clinic in Athens (a sample of convenience). The selection criteria were as follows: 1) age $>18$ years; 2 ) ability to communicate in Greek; 3) diagnosis of lung cancer; 4) receiving chemotherapy; and 5) adequate levels of cooperation and perceptive capacity. After the distribution of the questionnaires, 135 questionnaires were collected.

\section{Ethics}

This study was approved by the Ethics Committee of Athens Hospital for Chest Diseases "Sotiria", Athens, Greece. The patients were approached by a member of the research team and asked if they wanted to participate in the study. After they provided all the necessary information for the study, written consent was obtained from them. They were informed about the anonymity of the questionnaires and assured that the data would be used for research purposes only. This study was conducted in compliance with the ethical standards of the responsible institution on human subjects as well as with the Helsinki Declaration.

\section{Data collection tool}

A specially designed questionnaire consisting of the following parts was used to collect the data. The first part involved the demographic and clinical characteristics. The second was DASS21, which consists of 21 suggestions and three self-report scales designed to measure the negative emotional states of depression, anxiety and stress. The subscale of depression evaluates discomfort, despair, life underestimation and hypoestimation, lack of interest/participation, anhedonia and inertia. The stress subscale evaluates autonomic nervous stimulation as well as the effects of skeletal muscles, stressful conditions and subjective experiences of anxiety. This subscale is sensitive to the levels of chronic non-specific stimulation. It evaluates the difficulty of relaxing and nervous stimulation and if someone is easily upset and whether someone is acute/hyperreactive and impatient. Respondents respond through a fourpoint scale by assessing the extent to which they experienced each question in the last week [17]. The third part contained the health survey questionnaire of 12 questions, SF-12, which was developed as a shorter alternative to the SF-36 questionnaire; the latter is used in large-scale studies, especially when general physical and mental health are the results of interest rather than the characteristic diagram consisting of the eight scales of SF-36 [18]. All 12 questions have been used before to calculate the total physical and mental components (PCS-12 and MCS-12). The validity of the Greek version was evaluated in a study with a stratified representative sample $(n=1,005)$ of a healthy Greek population [19]. The data were initially analyzed based on descriptive statistics (frequencies, mean values and standard deviations) and inductive statistics to answer all the research questions. Inductive controls included the Spearman, variance analysis and independent sample tests. The significance level was set at $\mathrm{P}$ value of 0.05 (two-tailed).

\section{Results}

\section{Demographic and clinical characteristics}

Of the 135 patients, $69.6 \%$ were male. The mean age was 60.4 \pm 9.2 years, and $71.8 \%$ were married. From the sample, $39.3 \%$ were pensioners, $92.6 \%$ had a public insurer, $33.4 \%$ were elementary graduates and $32.6 \%$ were high-school graduates. Regarding the clinical characteristics of the sample, $25.2 \%$ suffered from small-cell cancer, $69.6 \%$ from non-small cell cancer and 5.2\% from large-cell cancer. The average duration of disease was 13.9 months, and $65.2 \%$ did not have any other health problems. The mean of hematocrit was $37.7 \pm 5.5$, hemoglobin was $13.3 \pm 4.4$ and white blood cell count was 8,332 $\pm 10,383$. Tables 1 and 2 contain details regarding the sample demographic and its clinical characteristics.

\section{QoL and psychological distress descriptive statistics}

As regards the QoL of our sample, we observed that the mean score of the physical health component of SF-12 was 38.17 \pm 9.94 and that of the mental health component was $45.63 \pm$ 11.80. As regards the psychological distress of our sample, we observed that the mean score for depression was $4.55 \pm 5.04$, for anxiety was $3.84 \pm 4.17$ and for stress was $5.21 \pm 5.01$. Details about the descriptive statistics related to the QoL and the psychological distress of lung cancer patients can be found in Table 3. Table 4 depicts the distribution of our sample among the categories of psychological distress, and we can observe that $35.6 \%$ were experiencing depressive symptomatology, $40 \%$ had symptoms of anxiety and $26.7 \%$ were undergoing stress. Upon bivariate examination, no statistically significant associations were found between the QoL and patients' demo- 
Table 1. Sociodemographic Characteristics of Participants (n = 135)

\begin{tabular}{|c|c|}
\hline \multicolumn{2}{|l|}{ Characteristics } \\
\hline \multicolumn{2}{|l|}{ Gender, N (\%) } \\
\hline Male & $94(69.6)$ \\
\hline Female & $41(30.4)$ \\
\hline Age (years), mean $\pm \mathrm{SD}$ & $60.4 \pm 9.2$ \\
\hline \multicolumn{2}{|l|}{ Family status, N (\%) } \\
\hline Unmarried & $21(15.6)$ \\
\hline Married & $93(68.9)$ \\
\hline Divorced & $5(3.7)$ \\
\hline Widow & $16(11.9)$ \\
\hline \multicolumn{2}{|c|}{ Living arrangements, N (\%) } \\
\hline Alone & $31(23)$ \\
\hline Cohabiting & $104(77)$ \\
\hline \multicolumn{2}{|c|}{ Occupational status, N (\%) } \\
\hline Retired & $53(39.3)$ \\
\hline Other & $32(23.7)$ \\
\hline Private employee & $29(21.5)$ \\
\hline Household & $9(6.7)$ \\
\hline State-employee & $7(5.2)$ \\
\hline Unemployed & $5(3.7)$ \\
\hline \multicolumn{2}{|l|}{ Educational level, N (\%) } \\
\hline Primary & $45(33.3)$ \\
\hline Secondary & $61(54.1)$ \\
\hline University & $17(12.6)$ \\
\hline
\end{tabular}

SD: standard deviation.

graphic and clinical characteristics.

\section{Association between the QoL and psychological distress}

The results of the Spearman correlation test indicated that there was a significant negative association between SF-12 physical health and depression $(\mathrm{r}(135)=-0.392, \mathrm{P}<0.001)$, anxiety $(\mathrm{r}(135)=-0.413, \mathrm{P}<0.001)$ and stress $(\mathrm{r}(135)=-0.398, \mathrm{P}$ $<0.001)$. Similar statistically significant negative correlations were observed between SF-12 mental health and depression $(\mathrm{r}(135)=-0.594, \mathrm{P}<0.001)$, anxiety $(\mathrm{r}(135)=-0.437, \mathrm{P}<$ $0.001)$ and stress $(\mathrm{r}(135)=-0.491, \mathrm{P}<0.001)$. A detailed report on the correlation between QoL and psychological distress is presented in Table 5.

\section{Discussion}

Lung cancer is a major public health problem in both developed and developing countries. This issue deserves further research so that better care and QoL can be provided to those
Table 2. Clinical Characteristics

\begin{tabular}{|c|c|}
\hline Characteristics & \\
\hline Other health problems, $\mathrm{N}(\%)$ & \\
\hline None & $88(65.2)$ \\
\hline One & $24(17.8)$ \\
\hline Two & $5(3.7)$ \\
\hline Three & $13(9.6)$ \\
\hline Four & $5(3.7)$ \\
\hline Type of lung cancer, N (\%) & \\
\hline Small cell & $34(25.2)$ \\
\hline Non-small cell & $94(69.6)$ \\
\hline Big cell & $7(5.2)$ \\
\hline Metastasis, N (\%) & \\
\hline Yes & $71(52.6)$ \\
\hline No & $64(47.4)$ \\
\hline Duration of illness (months), mean (SD) & $13.93(16.8)$ \\
\hline White blood cell count, $/ \mu \mathrm{L}$, mean (SD) & $8,332(10,383)$ \\
\hline Hemoglobin, g/dL, mean (SD) & $13.3(4.4)$ \\
\hline Hematocrit, \%, mean (SD) & $37.7(5.5)$ \\
\hline
\end{tabular}

SD: standard deviation.

with this disease. QoL refers to "global well-being" and includes the physical, emotional, mental, social and behavioral statuses of patients.

This study aimed at assessing the QoL of lung cancer patients receiving chemotherapy. It also explored the association between QoL and demographic and clinical characteristics as well as the relation between QoL and psychological distress. One important finding was that we did not observe any association between QoL and demographic and clinical characteristics. Moreover, according to our results, there is a significant negative relation between QoL and psychological distress.

The mean score of QoL for the physical health component of SF-12 was $38.17 \pm 9.94$ and for the mental health component was $45.63 \pm 11.80$. These scores are considered high when we compare them with similar studies on QoL in lung cancer [20]. In a study conducted in Greece, 95 advanced cancer patients' QoL was examined from a multidimensional perspective; the study reported high levels of physical function and QoL [21]. According to Polanski et al, lung cancer patients reported lower levels of QoL compared to not only healthy individuals but also patients suffering from other malignancies [22]. In a Greek study involving diabetic patients, even lower levels of QoL were reported, especially in the physical domain [23]. Patients with chronic diseases experience a range of symptoms, which vary in intensity and affect their QoL and, potentially, their functionality. The burdensome symptoms as well as other simultaneously occurring clinical variables are key factors that lead to low QoL in patients. The intensity and number of symptoms have been found to adversely affect several dimensions of QoL; this suggests the importance of effective symptom management in chronic diseases [24]. In a 
Table 3. Descriptive Statistics of SF-12 and DASS21

\begin{tabular}{llll} 
& Minimum value & Maximum value & Mean (SD) \\
\hline Physical health (SF-12) & 14.727 & 61.588 & $38.17(9.94)$ \\
Mental health (SF-12) & 18.947 & 67.646 & $45.63(11.80)$ \\
Depression & 0 & 21 & $4.55(5.04)$ \\
Anxiety & 0 & 19 & $3.84(4.17)$ \\
Stress & 0 & 21 & $5.21(5.01)$ \\
\hline
\end{tabular}

SD: standard deviation.

Table 4. Patients Distribution on Psychological Distress Categories $(n=135)$

\begin{tabular}{llllll} 
& Normal (I) & Mild (II) & Moderate (III) & Severe (IV) & Extremely severe (V) \\
\hline Depression & $87(64.4 \%)$ & $14(10.4 \%)$ & $16(11.9 \%)$ & $6(4.4 \%)$ & $12(8.9 \%)$ \\
Anxiety & $81(60 \%)$ & $21(15.6 \%)$ & $14(10.4 \%)$ & $9(6.7 \%)$ & $10(7.4 \%)$ \\
Stress & $99(73.3 \%)$ & $18(13.3 \%)$ & $4(3 \%)$ & $7(5.2 \%)$ & $7(5.2 \%)$ \\
\hline
\end{tabular}

large-scale study conducted in Greece in which 1,005 healthy individuals and individuals diagnosed with chronic diseases (diabetes, depression, hypertension and asthma) participated, the QoL and associated factors were examined using SF-12; finally, higher levels of QoL were reported [19]. Patients suffering from lung cancer face many difficulties due to the impact that the disease has on their functionality, the therapeutic approaches, their side effects and the mental burden caused by all these. All these factors ultimately make patients report poor QoL.

The role that demographic and clinical characteristics play in determining the QoL of lung cancer patients has been examined in several studies over the years. Most of them concluded that poor QoL is associated with lower socioeconomic status [25]. In our study, no association was found between QoL and demographic and clinical characteristics. This finding contradicts the findings of several studies conducted within the lung cancer research domain. According to Pierzynski et al, educational status, marital status and lung cancer type can influence the QoL of lung cancer patients [26]. Recently, a crosssectional study was conducted in Greece, and 200 lung cancer patients receiving chemotherapy had participated in it. It was found that cancer recurrence and educational status significantly affected the QoL of those patients [16]. This difference in the findings can be partly explained by taking into account the differences in the sample size and the setting in which the data were collected.
One more important finding of the present study is the prevalence of psychological distress among lung cancer patients. In our sample, $36.6 \%$ were experiencing depressive symptomatology, $40 \%$ showed symptoms of anxiety and $27.7 \%$ were undergoing stress. This finding is in agreement with and strengthens the results of other studies that point out that cancer-related suffering and disease-related symptoms cause patients emotional stress [27]. The percentages for the prevalence of psychological distress vary among many studies. For instance, in a recent multi-centre study involving 561 patients, the prevalence of depression and anxiety was and 19\% [28]. In another cross-sectional study, these percentages increased to $88 \%$ for depression and $71.5 \%$ for anxiety [29]. This variance can easily be explained by taking into consideration the differences in data collection methods, tools and settings. Additionally, several factors should be considered when interpreting data; for example, self-reported diagnosis lacks credibility, and cultural differences can cause differences in stress responses.

In the present study, a negative correlation was observed between QoL and psychological distress. More specifically, both the physical and mental components of SF-12 exhibited statistically significant negative correlations at the level of $\mathrm{P}<$ 0.001 . This result is in accordance with and reinforces the previous studies that reported a significant association between the symptoms of anxiety and depression and poor QoL [16, 3032]. Both psychological distress and poor QoL have been associated with adverse health outcomes in the cancer trajectory

Table 5. Correlation Between Quality of Life and Psychological Distress

\begin{tabular}{lllll}
\hline & I & II & III & IV \\
\hline SF-12 physical health (I) & & & & \\
SF-12 mental health (II) & 0.181 & & & \\
Depression (III) & $-0.392^{* *}$ & $-0.594 * *$ & $0.723 * *$ & $0.817 * *$ \\
Anxiety (IV) & $-0.413 * *$ & $-0.437 * *$ & $0.698 * *$ \\
Stress (V) & $-0.398 * *$ & $-0.491 * *$ & \\
\hline
\end{tabular}

${ }^{*} \mathrm{P}<0.05,{ }^{*} \mathrm{P}<0.001$, Spearman rho correlation coefficient. 
for several types of cancer [33-35]. This fact establishes the necessity of the implementation of interventions for reducing distress and promoting the QoL of cancer patients receiving chemotherapy [34].

There are some limitations of this study. The sample size is rather small $(\mathrm{n}=135)$, and participants were recruited only from one facility in Athens, Greece. Future studies must be conducted at the national level, so that the findings can be generalized.

\section{Conclusions}

Lung cancer is an important health issue that affects patients' QoL. In our study, lung cancer patients reported poor QoL and increased psychological distress. QoL was introduced as a tool for comparing cancer treatments, especially in the advanced stages of the disease. As an early indication of the progression of the disease, QoL could help doctors in daily practice as they closely monitor patients. QoL is affected by the patient's perception of illness and treatment, and it is altered by factors such as harm, stress, perceptions and social opportunities.

\section{Acknowledgments}

Authors would like to thank all patients for their participation in the study.

\section{Financial Disclosure}

None to declare.

\section{Conflict of Interest}

None to declare.

\section{Informed Consent}

Written consent was obtained from patients.

\section{Author Contributions}

PP, IVP and ECF conceived and designed the analysis. VB and PP collected the data. FM and DP performed the analysis. PP, VB and DP drafted the article. ECF, IVP and FM critically revised the manuscript. All authors reviewed and approved the final version of the manuscript.

\section{Data Availability}

The data supporting the findings of this study are available from the corresponding author upon reasonable request.

\section{References}

1. Kumar S, Saikia J, Kumar V, Jr., Malik PS, Madan K, Jain D, Bharati S. Neoadjuvant chemotherapy followed by surgery in lung cancer: Indian scenario. Curr Probl Cancer. 2020;44(3):100563.

2. Wang Q, Wang P, Zhang L, Tessema M, Bai L, Xu X, Li $\mathrm{Q}$, et al. Epigenetic regulation of RIP3 suppresses necroptosis and increases resistance to chemotherapy in nonsmall cell lung cancer. Transl Oncol. 2020;13(2):372-382.

3. Judd J, Borghaei H. Combining Immunotherapy and Chemotherapy for Non-Small Cell Lung Cancer. Thorac Surg Clin. 2020;30(2):199-206.

4. Kim R, Keam B, Hahn S, Ock CY, Kim M, Kim TM, Kim DW, et al. First-line pembrolizumab versus pembrolizumab plus chemotherapy versus chemotherapy alone in non-small-cell lung cancer: a systematic review and network meta-analysis. Clin Lung Cancer. 2019;20(5):331338 e334.

5. Rittberg R, Green S, Aquin T, Bucher O, Banerji S, Dawe DE. Effect of hospitalization during first chemotherapy and performance status on small-cell lung cancer outcomes. Clin Lung Cancer. 2020;21(5):e388-e404.

6. Akin S, Can G, Aydiner A, Ozdilli K, Durna Z. Quality of life, symptom experience and distress of lung cancer patients undergoing chemotherapy. Eur J Oncol Nurs. 2010;14(5):400-409.

7. Gralla RJ, Thatcher N. Quality-of-life assessment in advanced lung cancer: considerations for evaluation in patients receiving chemotherapy. Lung Cancer. 2004;46(Suppl 2):S41-47.

8. Wintner LM, Giesinger JM, Zabernigg A, Sztankay M, Meraner V, Pall G, Hilbe W, et al. Quality of life during chemotherapy in lung cancer patients: results across different treatment lines. Br J Cancer. 2013;109(9):23012308.

9. Gu W, Xu YM, Zhu JH, Zhong BL. Depression and its impact on health-related quality of life among Chinese inpatients with lung cancer. Oncotarget. 2017;8(62):104806104812.

10. Fischer IC, Cripe LD, Rand KL. Predicting symptoms of anxiety and depression in patients living with advanced cancer: the differential roles of hope and optimism. Support Care Cancer. 2018;26(10):3471-3477.

11. Yin S, Njai R, Barker L, Siegel PZ, Liao Y. Summarizing health-related quality of life (HRQOL): development and testing of a one-factor model. Popul Health Metr. 2016;14(22.

12. Wilson IB, Cleary PD. Linking clinical variables with health-related quality of life. A conceptual model of patient outcomes. JAMA. 1995;273(1):59-65.

13. Billis E, Koutsojannis C, Matzaroglou C, Gliatis J, Fousekis K, Gioftsos G, Papandreou M, et al. Association of low back pain on physical, sociodemographic and lifestyle factors across a general population sample within Greece. J Back Musculoskelet Rehabil. 2017;30(2):279290.

14. Wu YL, Fukuoka M, Mok TS, Saijo N, Thongprasert 
S, Yang JC, Chu DT, et al. Tumor response and healthrelated quality of life in clinically selected patients from Asia with advanced non-small-cell lung cancer treated with first-line gefitinib: post hoc analyses from the IPASS study. Lung Cancer. 2013;81(2):280-287.

15. Reck M, Taylor F, Penrod JR, DeRosa M, Morrissey L, Dastani H, Orsini L, et al. Impact of nivolumab versus docetaxel on health-related quality of life and symptoms in patients with advanced squamous non-small cell lung cancer: results from the checkmate 017 study. J Thorac Oncol. 2018;13(2):194-204.

16. Togas C, George A, Fotios A. Evaluation of health-related quality of life in lung cancer patients in Greece and associations with demographic and medical characteristics. Journal of Biosciences and Medicines. 2019;7(10):17-31.

17. Lyrakos GN, Arvaniti C, Smyrnioti M, Kostopanagiotou G. Translation and validation study of the depression anxiety stress scale in the Greek general population and in a psychiatric patient's sample. European Psychiatry. 2011;26(S2):1731.

18. Ware JE, Jr., Sherbourne CD. The MOS 36-item shortform health survey (SF-36). I. Conceptual framework and item selection. Med Care. 1992;30(6):473-483.

19. Kontodimopoulos N, Pappa E, Niakas D, Tountas Y. Validity of SF-12 summary scores in a Greek general population. Health Qual Life Outcomes. 2007;5:55.

20. Rendas-Baum R, D'Alessio D, Bjorner JB. Health-related quality of life predicted subsequent health care resource utilization in patients with active cancer. Qual Life Res. 2019;28(4):1085-1095.

21. Konstantinidis T, Linardakis M, Samonis G, Philalithis A. Quality of life of patients with advanced cancer treated in a regional hospital in Greece. Hippokratia. 2016;20(2):139-146.

22. Polanski J, Jankowska-Polanska B, Rosinczuk J, Chabowski M, Szymanska-Chabowska A. Quality of life of patients with lung cancer. Onco Targets Ther. 2016;9:1023-1028.

23. Georgios LN, Hatziagelaki E, Damigos D, Athanasia PK, Bousboulas S, Batistaki C. Predictors of health-related quality of life in Diabetic Neuropathy type II diabetic patients in Greece. Health Science Journal. 2013;7(3):327.

24. Fradelos E. Predictors of quality of life in chronic kidney disease: The contribution of spirituality. Archives of Hellenic Medicine. 2020;37(2):227-236.

25. Montazeri A, Hole DJ, Milroy R, McEwen J, Gillis CR. Quality of life in lung cancer patients: does socioeconom- ic status matter? Health Qual Life Outcomes. 2003;1:19.

26. Pierzynski JA, Ye Y, Lippman SM, Rodriguez MA, Wu $\mathrm{X}$, Hildebrandt MAT. Socio-demographic, clinical, and genetic determinants of quality of life in lung cancer patients. Sci Rep. 2018;8(1):10640.

27. Morrison EJ, Novotny PJ, Sloan JA, Yang P, Patten CA, Ruddy KJ, Clark MM. Emotional problems, quality of life, and symptom burden in patients with lung cancer. Clin Lung Cancer. 2017;18(5):497-503.

28. Eichler M, Hechtner M, Wehler B, Buhl R, Stratmann J, Sebastian M, Schmidberger H, et al. Psychological distress in lung cancer survivors at least 1 year after diagnosis-Results of a German multicenter cross-sectional study. Psychooncology. 2018;27(8):2002-2008.

29. Diaz-Frutos D, Baca-Garcia E, Garcia-Foncillas J, Lopez-Castroman J. Predictors of psychological distress in advanced cancer patients under palliative treatments. Eur J Cancer Care (Engl). 2016;25(4):608-615.

30. Chambers SK, Baade P, Youl P, Aitken J, Occhipinti S, Vinod S, Valery PC, et al. Psychological distress and quality of life in lung cancer: the role of health-related stigma, illness appraisals and social constraints. Psychooncology. 2015;24(11):1569-1577.

31. Ayley-Veloso R, Szabo YZ, Cash E, Zimmaro L, Siwik C, Kloecker G, Salmon P, et al. The association between history of traumatic events and health-related quality of life among lung cancer patients. J Psychosoc Oncol. 2020;38(5):627-634.

32. Pigkou K, Alikari V, Papathanasiou IV, Theophilou P, Lavdaniti M, Zyga S, Fradelos EC. Assessment of psychological distress and quality of life in lung cancer patients receiving chemotherapy: A single center experience. Progress in Health Sciences. 2018;8(1):126-33.

33. Muzzatti B, Bomben F, Flaiban C, Piccinin M, Annunziata MA. Quality of life and psychological distress during cancer: a prospective observational study involving young breast cancer female patients. BMC Cancer. 2020;20(1):758

34. Karunanithi G, Sagar RP, Joy A, Vedasoundaram P. Assessment of psychological distress and its effect on quality of life and social functioning in cancer patients. Indian J Palliat Care. 2018;24(1):72-77.

35. Al-Ghabeesh SH, Al-Kalaldah M, Rayan A, Al-Rifai A, Al-Halaiqa F. Psychological distress and quality of life among Jordanian women diagnosed with breast cancer: The role of trait mindfulness. Eur J Cancer Care (Engl). 2019;28(5):e13082. 